\title{
1 Tissue specific auxin biosynthesis regulates leaf vein patterning
}

2 Irina Kneuper ${ }^{1}$, William Teale ${ }^{1}$, Jonathan Edward Dawson ${ }^{2}$, Ryuji Tsugeki ${ }^{3}$, Klaus Palme ${ }^{1,4,5,6}$, Eleni

3 Katifori ${ }^{2,7} \&$ Franck Anicet Ditengou ${ }^{1}$

$4{ }^{1}$ Institute of Biology II, Faculty of Biology, Albert-Ludwigs-University of Freiburg, Schänzlestrasse 1,

579104 Freiburg, Germany.

6 2Physics of Biological Organization, Max Planck Institute for Dynamics and Self-Organization, Am

7 Fassberg 17, 37077 Göttingen, Germany.

$8{ }^{3}$ Department of Botany, Graduate School of Science, Kyoto University, Sakyo-ku, Kyoto 606-8502

9 Japan

$10{ }^{4}$ Center for Biological Systems Analysis, Albert-Ludwigs-University of Freiburg, Habsburgerstrasse 49,

1179104 Freiburg, Germany.

$12{ }^{5}$ Freiburg Institute for Advanced Sciences (FRIAS), Albert-Ludwigs-University of Freiburg,

13 Albertstrasse 19, 79104 Freiburg, Germany.

$14{ }^{6}$ BIOSS Center for Biological Signalling Studies, Albert-Ludwigs-University of Freiburg,

15 Schänzlestrasse 18, 79104 Freiburg, Germany.

$16{ }^{7}$ Department of Physics and Astronomy, University of Pennsylvania, PA, 19104, USA.

17

18 Corresponding Authors

19 Franck A. Ditengou (franck.ditengou@biologie.uni-freiburg.de). Twitter: @FDitengou

20 Klaus Palme (klaus.palme@biologie.uni-freiburg.de).

21 Lead Contact

22 Franck A. Ditengou (franck.ditengou@biologie.uni-freiburg.de) 


\section{Highlights}

25

The plant hormone auxin (indole-3-acetic acid, IAA) has a profound influence over plant cell growth

34 and differentiation. Current understanding of vein development in leaves is based on the canalization

35 of auxin into self-reinforcing streams which determine the sites of vascular cell differentiation.

36 However, the role of auxin biosynthesis during leaf development in the context of leaf vein patterning

37 has not been much studied so far. Here we characterize the context specific importance of auxin

38 biosynthesis, auxin transport and mechanical regulations in a growing leaf. We show that domains of

39 auxin biosynthesis predict the positioning of vascular cells. In mutants that have reduced capacity in

40 auxin biosynthesis, leaf vein formation is decreased. While exogenous application of auxin does not

41 compensate the loss of vein formation in auxin biosynthesis mutants, inhibition of polar auxin

42 transport does compensate the vein-less phenotype, suggesting that the site-specific accumulation

43 of auxin, which is likely to be mainly caused by the local auxin biosynthesis, is important for leaf vein

44 formation. Our computational model of midvein development brings forth the interplay of cell

45 stiffness and auxin dependent cell division. We propose that local auxin biosynthesis has the integral

46 role in leaf vascular development.

$48 \quad$ Keywords

49 Auxin, leaf, vein patterning, mathematical modelling 


\section{Highlights and eTOC Blurb}

51 Using modelling and a spatiotemporal analysis of auxin biosynthesis and transport, Kneuper et al.

52 show that tissue specific auxin biosynthesis defines places of vein initiation hence underlining the

53 importance of auxin concentration in vein initiation.

\section{Introduction}

Vascular systems are continuous networks of cells which connect a wide range of tissues. In leaves,

57 veins form characteristic patterns which support photosynthesis in the surrounding mesophyll cells.

58 However, despite leaf venation patterning being important for the overall fitness of the plant (Blonder

59 et al., 2011), the processes which guide vein placement are not well understood. In plants the eventual

60 pattern of the vascular network is widely believed to be controlled by the phytohormone auxin (Bruck

61 and Paolillo, 1984), via its canalization into self-reinforcing streams (Effendi et al., 2015; Sachs, 1969).

62 Support for with-the-flux-based explanations of leaf vascular development followed the identification

63 of PIN1: a long-predicted auxin efflux protein and the earliest marker of leaf vascular cell identity

64 (Galweiler et al., 1998; Scarpella et al., 2006). The polar localization of PIN1 in leaf epidermal cells has

65 offered a mechanism which focuses auxin to specific epidermal domains which correlate with the

66 directions of future vein development (Scarpella et al. 2006).

67 Experimental studies on auxin canalization have been corroborated with theoretical models based on

68 the auxin canalization hypothesis, that have shown how reinforcing fluxes in a fixed domain of leaf

69 tissue can create channels of auxin that resemble the vein patterns observed in leaves (Rolland-Lagan

70 and Prusinkiewicz, 2005). However, the robustness of canalization models is reduced when applied to

71 a growing tissue (Feller et al., 2015; Lee et al., 2014). The canalization hypothesis states that the

72 position of auxin sources in the leaf primordium is critical for vein formation. It has been proposed

73 that these sources are derived from the focal points of epidermal auxin flux, from where auxin flows 
74 in channels towards the base of the leaf (Scarpella et al., 2006). However, in addition to epidermis-

75 derived auxin (Abley et al., 2016), auxin is also synthesized in the lamina of the growing primordium;

76 an observation which has, to date, not been considered by any models. In support of a major role for

77 auxin biosynthesis in vascular development in leaves, Cheng and colleagues observed progressively

78 fewer and more discontinuous vascular strands when less auxin is synthesized in the primordium

79 (Cheng et al., 2006), suggesting that local biosynthesis may lift auxin concentration above certain

80 thresholds required for vascular tissues to form.

81 In this study, which combines a series of experiments with theory and assesses the role of auxin

82 biosynthesis in vein initiation and patterning in the growing leaf, we find that leaf vein initiation is

83 strongly correlated with the expression domains of auxin biosynthetic enzymes within primordia. We

84 build an auxin-transport-independent model of vascular tissue development, and use it to identify cell

85 expansion, auxin production rate, cellular auxin concentration and the auxin concentration-dependent

86 cell growth of non-auxin producing cells as a robust, minimal set of parameters, for spontaneous

87 organization of primordium cells into a midvein.

\section{Results}

90 Reducing a plant's capacity to synthesize auxin, either genetically or by the application of enzyme

91 inhibitors, impairs the development of leaf vein networks (Nishimura et al., 2014; Stepanova et al.,

92 2008). In mutants defective in auxin biosynthesis, leaves have fewer, more widely spaced veins which

93 are often disorganized and discontinuous (Figures $1 \mathrm{~A}$ and S1A-S1E, S1H). We therefore first wanted

94 to ascertain whether auxin biosynthesis acts exclusively through increasing the overall amount of auxin

95 available for transport-based patterning mechanisms. However, exogenous application of auxin cannot

96 rescue aberrant vein development in wei8-1tar2-1, a mutant defective in auxin biosynthesis 
97 (Stepanova et al., 2011) (Figure 1), suggesting that it is not simply the amount of auxin produced which

98 is important, but that the site of auxin production is also important for vein formation.

99 Two families of enzymes act in series to catalyze the indole-3-pyruvic acid (IPA)-dependent production

100 of IAA: tryptophan aminotransferases (TAA1/WEI8, TAR1 and TAR2) and YUCCA flavin

101 monooxygenases (Mashiguchi et al., 2011; Stepanova et al., 2011; Zhao, 2012). Here, we focused on

102 YUC1, YUC2, YUC4, YUC6, as they are the main YUCs for auxin biosynthesis in shoots, whereas the

103 other YUCs (i.e. YUC3, YUC5, YUC7, YUC8, YUC9) are responsible for producing auxin in roots (Won et

104 al., 2011; Zhao, 2012). In order to resolve spatial synthesis of auxin within the lamina of a growing leaf,

105 we visualized the expression pattern of auxin biosynthetic genes and their correlation with pre-

106 provascular cells in the leaf primordium. We built an auxin-biosynthesis map in Arabidopsis, at cellular

107 resolution using well established standard techniques (imaging of GFP fusion proteins, promoter GUS

108 fusions), which reflect faithfully the expression of auxin biosynthetic genes in leaf primordia over time.

109 At between 2 and 5 days after germination (DAG), enzymes of YUCCA and TAA1 families showed highly

110 localized expression patterns in which the future sites of vascular cells can be clearly seen (Figures 2;

111 S2 and S3). Over this time period, expression domains of both classes of enzymes were restricted to

112 the regions of leaf primordia in which veins are formed, and the border between the abaxial and

113 adaxial sides (lower panel in Figure 2A and Figure S3). TAA-type aminotransferases and YUCCA genes

114 are expressed in distinct as well as in overlapping domains (Figures 2, S2 and S3). Note particularly

115 the overlapping expression of TAA1, TAR2, and YUC4 during the early stage of midvein development

116 in 2 day-old primordia (Figures 2, S2 and S3). Since TAA and YUC act in succession in the same IPA

117 pathway to produce IAA from tryptophan, co-expression of TAA and YUC in the same cells increases

118 the chance to produce IAA.

119 The effect of inhibiting polar auxin transport on vein patterning, either by introducing genetic lesions

120 (Okada et al., 1991), or by the exogenous application of diverse auxin transport inhibitors (Mattsson 
121 et al., 1999; Sieburth, 1999), has been known for decades. The inhibition of auxin transport, either

122 genetically or by the application of inhibitors, leads to the formation of small, round leaves with an

123 indistinct midvein and fused vascular bundles around the periphery of their distal end (Mattsson et al.,

124 2003; Mattsson et al., 1999). Therefore, it was proposed that in a developing Arabidopsis leaf, auxin

125 transport defines the localization of leaf veins (Scarpella et al., 2006). In order to ascertain whether

126 auxin transport also defines sites of auxin biosynthesis, we examined the distribution of cells which

127 contained auxin biosynthetic enzymes (or their transcripts) in the leaf primordium after inhibition of

128 auxin efflux.

129 In Arabidopsis, 1-N-naphthylphthalamic acid (NPA) abolishes polar auxin transport at concentrations

130 above $10 \mu \mathrm{M}$ (Thomson et al., 1973). After treatment with increasing concentrations of NPA up to 320

$131 \mu \mathrm{M}$, leaves became round and gradually smaller (Figure 3A). Furthermore, they developed an increased

132 number of secondary veins and an exaggerated mesh of fused tracheary elements which surrounded

133 the leaf margin, when compared to leaves of untreated plants (Cai et al., 2014) (Figure 3A). After

134 blocking auxin transport, the domains of auxin biosynthetic enzymes expanded and continued to

135 predict the regions of vein initiation (Figures 3B-3E, S4). In leaf lamina, with or without NPA treatment,

136 PIN1 expression in the lamina was confined to cells which lay within auxin biosynthetic domains

137 (Figure 3C-3F). The change in shape of the leaf after NPA treatment is correlated with the proliferation

138 of provascular cells expressing both PIN1 and auxin biosynthetic genes in these leaves (Figure 3B-3F).

139 In summary, NPA application changed the auxin biosynthesis pattern, but veins were nevertheless still

140 formed in regions of auxin biosynthesis [Fig. S4A (lower panel), S4B and S4C]. Therefore, blocking

141 auxin efflux causes auxin levels in non-vascular cells to drop, which then lowers cell division rates in

142 the non-vascular cells causing vascular cells to proliferate laterally (Figure 3B-3F). NPA also induced

143 the accumulation of auxin at the distal end of the leaf (as indicated by DR5::Venus in Figure $3 \mathrm{H}$,

144 compare upper versus lower panel), suggesting the distal sites also produce auxin (Abley et al., 2016;

145 Avsian-Kretchmer et al., 2002). 


\section{In silico modeling of spontaneous patterning of vascular cells in the leaf primordium}

147 In order to test whether cell specific auxin biosynthesis and its impact on neighboring cells is able to

148 drive leaf vein patterning, we developed a theoretical model and performed a series of in silico

149 experiments. We adapted Virtual Leaf, an open-source cell-based modeling framework that describes

150 cells of the leaf lamina as a two-dimensional layer of interconnected polygons and accounts for

151 mechanical properties of the tissue (Merks et al., 2011). Cell growth (an irreversible increase in cell

152 area) proceeds in a quasi-static way at a rate which is defined by cell turgor pressure, a feature that

153 can be influenced by auxin (see Methods section). A cell divides each time its area doubles, which

154 invariably results in tissue growth over time. Cell wall stiffness and mechanics are included in the

155 model by the definition of a resting length for each cell wall element. The shape of the tissue at each

156 stage of growth is determined by minimizing the generalized energy function that contains a cell area

157 term and a cell wall elasticity term (see computational model description in the Methods section).

158 To model leaf vein patterning in a growing leaf, we created a leaf tissue template that closely resembles

159 a 2-day-old leaf primordium as our initial condition (Figure 4A). Following Lee, Feugier et al. (2014)

160 and Merks, Guravage et al. (2011), we modelled primary vein development in two dimensions. The

161 model tissue template consisted of cells expressing auxin biosynthesis genes, labeled as auxin

162 producer cells, and cells that do not synthesize auxin. In the model, auxin producer cells correspond

163 to the cells that express the auxin biosynthetic gene TAR2, and their layout in the tissue resembles the

164 TAR2-driven GUS expression in 2-day-old control leaf-primordium (Figure 4A). The mechanical

165 constraint due to the attachment of the leaf to the meristem is modeled by including a row of cells at

166 the proximal part of the leaf (the petiole). These cells also act as auxin sinks, to model auxin drainage

167 from the leaf via established veins at the stem (see computational model description in the Methods

168 section). Further assumptions of our model are as follows: i) inter-celullar auxin transport via a

169 diffusion-like process (PIN1 has a non-polar distribution in pre-provascular cells), ii) every cell in the

170 leaf lamina grows by increasing its area at the same rate, iii) auxin is known to promote cell expansion 
171 in aerial tissues (Fendrych et al., 2016; Perrot-Rechenmann, 2010), therefore when auxin concentration

172 in a non-auxin producing cell crosses a threshold, then this cell starts expanding at a higher rate, iv)

173 auxin-synthesising cells grow at all times at the basic rate (as defined in (ii)), and v) a relatively stiff

174 epidermal cell layer, as it is known that leaf epidermal cells are stiffer than laminar cells (Onoda et al., 175 2015).

176 Simulations of this simple model, using the experimentally reported values of various parameters (see

177 computational model description, Table S1) (Mitchison 1980), generated an in silico leaf-primordium

178 that had grown to a size of either approximately 150 cells (Figures 4B) or 900 cells (Figure S5). This

179 simulation result showed that, for a wide range of parameter values determining auxin-dependent cell

180 growth (see computational model description in the Methods section), and without including

181 polarized auxin transport, this model is able to reproduce i) proliferation of auxin synthesizing cells,

182 ii) correct midvein positioning and iii) coordinated vascular cell elongation, as experimentally observed

183 (Figures 4B-4C; S5 and Video S1). The shape of midvein generated by the model is robust with respect

184 to different initial geometry and number of auxin synthesizing cells (Fig. 4A left and right panel and

185 Fig. S7). Our simulations showed that localized auxin synthesis, followed by auxin diffusion, was able

186 to account for the establishment of a time-dependent auxin gradient across the tissue of leaf

187 primordium (inset of Figure 4B). Such an auxin gradient caused cells to grow at different growth rates.

188 This in turn induced transverse forces acting laterally on the walls of midvein cells. These forces are

189 are much larger in magnitude than the forces acting on other cells in the leaf lamina (Figure 4B). These

190 forces exerted by the neighboring cells on the midvein cells, resulted in non-trivial strain and force

191 distributions in the tissue that prevented the midvein cells from proliferating and thus resulted in the

192 development of a thin vascular strand of elongated cells as is characteristic of procambial cells

193 (Scarpella et al., 2006). 
194 We next studied in silico, the impact of reducing auxin transport rates on midvein formation. An NPA-

195 dependent reduction in auxin efflux led to a change in leaf vascular patterning and an expansion of-

196 the TAR2 expressing domain, suggesting an extension of auxin biosynthesis sites (Figure 4E).

197 Simulations of our model showed that a 25 -fold lowering of the rate of auxin transport altered the

198 formation of the midvein, and resulted in much wider proliferation of auxin-producing cells, as

199 observed in experiments (Figure 4D). Reducing auxin transport rate led to higher auxin concentrations

200 in auxin producer cells and steeper auxin gradients in the primordia (Figure $3 \mathrm{H}$ lower panel and inset

201 of Figure 4D), with non-auxin producing cell receiving less auxin from auxin producing cells and hence,

202 expanding in that case less than in leaves not treated with NPA. As a result, the auxin-dependent forces

203 exerted by the neighboring cells on the midvein cells were not strong enough to prevent the

204 proliferation of auxin producer cells (Figure 4D). The model predicted a midvein, several cells wide

205 with a high auxin concentration, just as was observed in plants grown on inhibitory concentrations of

206 NPA (Figures 3C-3E, 3F-3G, 3H and 4E). Furthermore, the midvein cells of the "NPA-treated" model

207 leaf did not elongate in the same way as "untreated" simulations (Figure 4D and inset, and Video S2).

208 Compensation of vein patterning in auxin biosynthesis mutants by NPA

209 The model predicted that NPA effects were due to the accumulation of auxin in developing vascular

210 cells. We therefore predicted that the reduced-efflux phenotype would be rescued by simultaneously

211 decreasing the rate of auxin synthesis. Accordingly, we simulated the treatment of auxin biosynthetic

212 mutants with NPA by simultaneously reducing both the auxin transport rate and the auxin production

213 rate (2-fold). Under a range of conditions, wild-type leaf vascular patterning was restored, but only

214 when the overall cell area growth rate was decreased (Figures 4F, S6A-D) (Methods section). In planta,

215 the sequential removal of auxin biosynthetic enzymes resulted in the differentiation of fewer vascular

216 strands, or even in the complete inhibition of vein development (Figures 5C, left panel and S1A-E).

217 However, as predicted by the model, many of these phenotypes were abrogated by the application of 
218 NPA (Figure 5C and 5D). This observation suggests that the cellular auxin concentration per se can

219 define vascular cell differentiation in leaf primordia. We therefore conclude that an underlying pattern

220 of auxin biosynthesis, influenced by the physical environment of the primordium, can direct vascular

221 cell initiation.

\section{In silico modeling of PIN1 based auxin transport}

223 Polar auxin transport has long been considered a major driver of leaf vascular cell differentiation

224 (Figure S1F) (Scheres and Xu, 2006). "With the flux" models of leaf vascular patterning assume that 225 cells sense the overall rate of auxin flux and respond by reinforcing this flux with the polar deposition

226 of PIN proteins. We therefore studied computationally the effect of polar auxin transport on the

227 formation of the midvein in the presence of localized auxin biosynthesis (see Methods section) (Figure

228 4G). Our simulation results show that polar auxin transport reproduces the midvein in much the same

229 way as in the diffusion-only case (discussed above; Figure 4B), suggesting that these two patterning

230 mechanisms may be linked or working in parallel.

\section{Regulation of auxin biosynthesis by auxin}

232 As the canalization of auxin requires a positive feedback loop between the plasma membrane's

233 permeability to auxin and the rate of auxin flux (Mitchison, 1981), we next addressed whether auxin

234 biosynthesis and flux participate at a transcriptional level in such a positive feedback loop. Mutants

235 defective in auxin biosynthesis were examined for PIN1 expression. However, the polarization of PIN1

236 proceeded as normal in five-day old leaves of wei8-1tar2-1 and yuc1yuc4 loss-of-function plants,

237 despite a drastic reduction in the density of high order veins being observed (Figures 6B and 6C, lower

238 panel of S1B, S1E and S1H). Next, neither TAA/TARs nor YUCCA transcription is induced by IAA (Figure

239 6D). This experiment was performed three times with similar results. Verification in Genvestigator

240 (www.genevestigator.com), also confirmed that the expression of TAA1, TAR1, TAR2, YUC1, YUC2, YUC4

241 and YUC6 genes is not induced by auxin. Further, our results also indicate that YUC1, YUC2, YUC4 and 
242 YUC6 are repressed by auxin; similar results were reported by Suzuki and colleagues (Suzuki et al.,

243 2015). Finally, we found that TAA1 expression was reduced as PIN1 accumulated and polarized in

244 provascular cells (Figure 7B, 7C). IAA biosynthesis is therefore not induced by the canalization of IAA,

245 but is likely to be integrated into an independent upstream patterning mechanism, as although TAA1

246 was seen in domains in which PIN1 is absent (Figure 7D-7I); the opposite case was never observed.

247 These observations suggest that auxin biosynthesis is not part of the positive feedback loop proposed

248 to initiate vasculature development in leaves.

\section{Discussion}

250 In plants, polarized auxin transport is crucial for the initiation and regulation of several developmental

251 programs. PIN-mediated auxin flux is widely accepted to be responsible for vascular patterning in

252 leaves, with vascular differentiation occurring along axialized (canalized) auxin streams (Scarpella et

253 al., 2006; Sieburth, 1999). Here we provide evidence that vein initiation also requires tissue specific

254 local auxin biosynthesis. This supported by the fact that in mutants defective in local auxin

255 biosynthesis, vein density is severely reduced (Cheng et al., 2006; Stepanova et al., 2008).

256 In leaf primordia, auxin transport does not only occur in developing veins, but also in epidermal cells

257 (Abley et al., 2016). Blocking auxin transport results in smaller, round leaves which display a 258 characteristic venation pattern. It was shown that cells accumulate more IAA when treated with NPA

259 (Petersson et al., 2009; Petrasek et al., 2006). Therefore, we hypothesized that, since auxin is synthesized

260 in both vein cells and epidermis cells, applying NPA would increase auxin concentration in both areas.

261 This did not lead to an increase in leaf size, suggesting that auxin does not increase the rate of cell

262 division in the epidermis. Similarly in roots, auxin induces cell division in pericycle cells, but not in 263 epidermal cells (Himanen et al., 2002; Mähönen et al., 2014; Pacheco-Villalobos et al., 2016). Thus, in

264 presence of NPA, epidermal cells (less sensitive to auxin) would divide less and as they surround 
265 proliferating vascular cells in the lamina, this would supply a possible reason for the change in 266 mechanical properties of the leaf identified by our model.

267 It was shown that in wild-type leaves, midvein provascular cells have potential characters of midvein 268 progenitor cells (Tsugeki et al., 2009). This observation is consistent with a hypothesis that clonal 269 populations of vein cells originate from those cells of the primordium which already have a vascular 270 identity. This hypothesis is supported by an experiment using the expression of CRE recombinase 271 under the control of the heat shock promoter (Ichihashi et al., 2011) which showed that in developing 272 leaf, clonal sectors give rise to connected veins.

273 The patterning of organ growth and development is constrained and directed by the cells' physical 274 environment, with mechanical stresses in particular influencing a broad range of developmental 275 pathways (Sampathkumar et al., 2014). Microtubules responsively re-orientate their direction 276 according to the stress landscape of a tissue in order to reinforce cells against the strains caused by 277 directional growth (Hamant et al., 2008). Considering the pervasive influence of mechanical stress over 278 plant development and patterning, it is likely that they also play a role in developing leaf primordium.

279 Our model is able to explain aspects of leaf development which include the production of auxin by 280 pre-provascular cells of young leaves, the presence of vascular tissue in leaves treated with high 281 concentrations of NPA (Mattsson et al., 1999), the relative insensitivity of vascular cell initiation to NPA 282 when compared to a reduction in auxin biosynthesis, and the strong auxin response maxima observed 283 in differentiating vascular cells (Bayer et al., 2009; Heisler et al., 2005). Using the model we present 284 here, we were able to recreate faithfully four key features of vascular cell biogenesis in the absence of 285 polar auxin transport: vascular strand formation, midvein positioning and coordinated vascular cell 286 elongation. Our analysis suggests a critical role for the site of auxin biosynthesis in directing vascular 287 cell differentiation. Thus, local auxin biosynthesis and a correspondingly high cellular auxin 288 concentration need to be considered alongside directional auxin flux as an important factor defining 
289 leaf vascular cell patterning. This conclusion is supported by the requirement for context-specific auxin

290 biosynthesis to complement multiple yucca mutants (as opposed to exogenous application) (Cheng

291 et al., 2006) and the fact that relatively small reductions in auxin amounts at a whole-plant level can

292 cause surprisingly severe auxin-deficient phenotypes (Stepanova et al., 2011).

293 The complete sequence of genes necessary auxin biosynthesis is expressed in the leaf lamina

294 (Mashiguchi et al., 2011; Stepanova et al., 2011; Zhao, 2012). Besides TAA1 whose expression in pre-

295 provascular cells extends to regions that do not make veins and then is downregulated once pre-

296 provascular cells become provascular cells, TAR2 is expressed exclusively in regions that make veins,

297 where YUC2 and YUC4 are also expressed. This highlights an established correlation between auxin

298 biosynthesis and vein development: in mutants defective in auxin biosynthesis, PIN1 expression and

299 high-order vein density decrease. In contrast, suppression of auxin transport in pin mutants or using

300 chemical auxin transport inhibitors has only a marginal impact on vein initiation. This suggests that

301 auxin biosynthesis may act upstream auxin transport and is necessary for leaf vein initiation. According

302 to the auxin transport-based canalization hypothesis, it is the auxin flux (rate of auxin molecules going

303 through the membrane in a polar PIN1-dependent manner) that initiates veins. On the other hand,

304 leaf veins are still formed when polar auxin transport is chemically abolished (as when a seedling is

305 treated with NPA, 2,3,5-triiodobenzoic acid (TIBA) or any other auxin transport inhibitor) (Carland et

306 al., 2016), which is hard to be explained only by the auxin transport-based canalization hypothesis.

307 Together these observations underline the crucial role of tissue specific auxin biosynthesis which may

308 act prior auxin canalization.

309 Our model predicted that reducing auxin biosynthesis and cell growth rates in the presence of NPA

310 would restore normal venation patterns in wei/ tar mutants. Indeed, treating auxin biosynthesis

311 mutants, which display both reduced auxin levels and growth rates (they develop smaller leaves) with

312 NPA, did consistently restore wild-type like vein patterning. Since cells accumulate more IAA when 
313 treated with NPA (Keller et al., 2004; Petersson et al., 2009; Petrasek et al., 2006), the abnormal venation

314 pattern in weil tar mutants is probably due to cell specific decreases in auxin concentration. Therefore,

315 the inhibition of auxin efflux by NPA would result in an increase of auxin concentration in pre-

316 /provascular cells, which would then trigger the initiation of venation. However, since NPA also affects

317 the shape of the leaf, it confirms that auxin transport in the epidermal cells plays an important role in

318 maintaining leaf shape as previously proposed (Izhaki and Bowman, 2007; Scarpella et al., 2010).

319 Taking into account cell auxin biosynthesis in the leaf lamina and auxin transport in epidermal cells in

320 future models will enable us to understand how leaf shape and vein patterning are coordinated.

321 In leaf lamina, auxin is transported through elongating vascular cells. However, it is not likely that auxin

322 transport in elongating vascular cells is a prerequisite for midvein formation or vascular branching,

323 since veins still form in presence of very high concentrations of NPA. Instead, the model predicts that

324 mechanical forces exerted on the cells synthesizing auxin; forces which would be sufficient to direct

325 the development of vascular strands. Indeed, the importance of geometrical and mechanical

326 constraints during vascular tissue development in the Arabidopsis embryonic root has already been

327 underlined (De Rybel et al., 2014). Our data show that unlike PIN1, whose expression is clearly

328 stimulated by auxin, biosynthetic genes are either insensitive to, or repressed by auxin. Moreover, i)

329 TAA1 expression is reduced, not increased, as PIN1 accumulates and polarizes in provascular cells; ii)

330 PIN1 expression is strongly down-regulated in leaves of auxin biosynthesis mutants; and iii) whilst

331 TAA1 can be seen in domains in which PIN1 is absent, the opposite case is never observed. Taken

332 together, these observations suggest that local auxin biosynthesis in leaf primordia is not part of the

333 auxin canalization positive feedback loop and plays an integral role in leaf vasculature development.

334 So far our model only focuses on the development of the midvein. The initial branching stages to

335 secondary and tertiary veins is likely to require more complex models, which are outside the scope of

336 this work. The search for factors which drive vascular patterning needs urgently to be redirected to 
337 include the highly complicated patterns of auxin biosynthesis. The regulation of auxin biosynthesis by

338 specific transcription factors (Cui et al., 2013) may complete such a mechanism.

\section{Author Contributions}

340 F.A.D., W.D.T., I.K. and K.P. conceived and designed the experiments. F.A.D., I.K. performed the

341 experiments. J.D. performed the mathematical modeling. F.A.D., I.K., W.D.T., E.K., R.T. and K.P. analyzed

342 the data. F.A.D., W.D.T, E.K. and K.P. wrote the paper. All authors discussed the results and commented

343 on the manuscript. Correspondence and request for material should be addressed to F.A.D.

344 (franck.ditengou@biologie.uni-freiburg.de) and K.P. (klaus.palme@biologie.uni-freiburg.de).

\section{Acknowledgements}

346 This work could not have been accomplished without the help of colleagues, collaborators and friends

347 who provided support, suggestions and materials. Particularly we would like to thank Jose Alonso and

348 Yunde Zhao for sharing materials. We also gratefully acknowledge the excellent technical support

349 from Beata Ditengou and Katja Rapp.

350 This work was supported by the Baden-Württemberg Stiftung, Deutsche Forschungsgemeinschaft (SFB

351 746), the Excellence Initiative of the German Federal and State Governments (EXC 294),

352 Bundesministerium für Forschung und Technik (BMBF SYSTEC, PROBIOPA, MICROSYSTEMS),

353 Deutsches Zentrum für Luft und Raumfahrt (DLR 50WB1022), the Freiburg Initiative for Systems

354 Biology, the European Union Framework 6 Program (AUTOSCREEN, LSHG-CT-2007-037897), the

355 National Science Foundation (USA), and JSPS KAKENHI (Grant Number 24570047, 16K07396). EK

356 acknowledges support from the Burroughs Wellcome Fund. 
361

362

363

364

365

366

367

368

369

370

371

372

373

374

375

376

377

378

379

380

381

382

383

384

385

386

387

388

389

390

391

392

393

394

395

396

397

398

399

400

401

402

403

404

405

Abley, K., Sauret-Gueto, S., Maree, A.F.M., and Coen, E. (2016). Formation of polarity convergences underlying shoot outgrowths. Elife 5. https://doi.org/10.7554/eLife.18165.001.

Avsian-Kretchmer, O., Cheng, J.C., Chen, L.J., Moctezuma, E., and Sung, Z.R. (2002). Indole acetic acid distribution coincides with vascular differentiation pattern during Arabidopsis leaf ontogeny. Plant Physiology 130, 199-209. DOI: http://dx.doi.org/10.1104/pp.003228.

Bayer, E.M., Smith, R.S., Mandel, T., Nakayama, N., Sauer, M., Prusinkiewicz, P., and Kuhlemeier, C. (2009). Integration of transport-based models for phyllotaxis and midvein formation. Genes \& Development 23, 373-384. DOI: 10.1101/gad.497009.

Blonder, B., Violle, C., Bentley, L.P., and Enquist, B.J. (2011). Venation networks and the origin of the leaf economics spectrum. Ecol Lett 14, 91-100. DOI: 10.1111/j.1461-0248.2010.01554.x.

Bruck, D.K., and Paolillo, D.J. (1984). Replacement of leaf primordia with IAA in the induction of vascular differentiation in the stem of Coculus. New Phytologist 96, 353-370.

Cai, X.T., Xu, P., Zhao, P.X., Liu, R., Yu, L.H., and Xiang, C.B. (2014). Arabidopsis ERF109 mediates crosstalk between jasmonic acid and auxin biosynthesis during lateral root formation. Nature Communications 5, 5833. DOI: 10.1038/ncomms6833.

Carland, F., Defries, A., Cutler, S., and Nelson, T. (2016). Novel Vein Patterns in Arabidopsis Induced by Small Molecules. Plant Physiology 170, 338-353. DOI: 10.1104/pp.15.01540.Cheng, Y., Dai, X., and Zhao, Y. (2006). Auxin biosynthesis by the YUCCA flavin monooxygenases controls the formation of floral organs and vascular tissues in Arabidopsis. Genes Dev 20, 1790-1799. DOI: 10.1101/gad.1415106.

Cui, D., Zhao, J., Jing, Y., Fan, M., Liu, J., Wang, Z., Xin, W., and Hu, Y. (2013). The arabidopsis IDD14, IDD15, and IDD16 cooperatively regulate lateral organ morphogenesis and gravitropism by promoting auxin biosynthesis and transport. PLoS Genet 9, e1003759. DOI: 10.1371/journal.pgen.1003759.

De Rybel, B., Adibi, M., Breda, A.S., Wendrich, J.R., Smit, M.E., Novak, O., Yamaguchi, N., Yoshida, S., Van Isterdael, G., Palovaara, J., et al. (2014). PLANT DEVELOPMENT Integration of growth and patterning during vascular tissue formation in Arabidopsis. Science 345. DOI:10.1126/science.1255215.

Ditengou, F.A., Teale, W.D., Kochersperger, P., Flittner, K.A., Kneuper, I., van der Graaff, E., Nziengui, H., Pinosa, F., Li, X., Nitschke, R., et al. (2008). Mechanical induction of lateral root initiation in Arabidopsis thaliana. Proceedings of the National Academy of Sciences of the United States of America 105, 1881818823. DOI: $10.1073 /$ pnas. 0807814105.

Effendi, Y., Ferro, N., Labusch, C., Geisler, M., and Scherer, G.F. (2015). Complementation of the embryolethal T-DNA insertion mutant of AUXIN-BINDING-PROTEIN 1 (ABP1) with abp1 point mutated versions reveals crosstalk of ABP1 and phytochromes. Journal of Experimental Botany 66, 403-418. DOI: 10.1093/jxb/eru433.

Feller, C., Farcot, E., and Mazza, C. (2015). Self-Organization of Plant Vascular Systems: Claims and Counter-Claims about the Flux-Based Auxin Transport Model. PLoS One 10. DOI: 10.1371/journal.pone.0118238.

Fendrych, M., Leung, J., and Friml, J. (2016). TIR1/AFB-Aux/IAA auxin perception mediates rapid cell wall acidification and growth of Arabidopsis hypocotyls. Elife 5. DOI: 10.7554/eLife.19048.

Galweiler, L., Guan, C., Muller, A., Wisman, E., Mendgen, K., Yephremov, A., and Palme, K. (1998). Regulation of polar auxin transport by AtPIN1 in Arabidopsis vascular tissue. Science 282, 2226-2230. Gibson, L.J., Ashby, M.F., and Easterling, K.E. (1988). Structure and Mechanics of the Iris Leaf. J Mater Sci 23, 3041-3048. DOI: 10.1016/j.jbiomech.2004.09.027.Hamant, O., Heisler, M.G., Jonsson, H., Krupinski, P., Uyttewaal, M., Bokov, P., Corson, F., Sahlin, P., Boudaoud, A., Meyerowitz, E.M., et al. (2008). Developmental Patterning by Mechanical Signals in Arabidopsis. Science (Washington D C) 322, 1650 1655. DOI: 10.1126/science.1165594. 
406

407

408

409

410

411

412

413

414

415

416

417

418

419

420

421

422

423

424

425

426

427

428

429

430

431

432

433

434

435

436

437

438

439

440

441

442

443

444

445

446

447

448

449

450

451

452

453

Heisler, M.G., Ohno, C., Das, P., Sieber, P., Reddy, G.V., Long, J.A., and Meyerowitz, E.M. (2005). Patterns of auxin transport and gene expression during primordium development revealed by live imaging of the Arabidopsis inflorescence meristem. Curr Biol 15, 1899-1911. DOI: 10.1016/j.cub.2005.09.052

Himanen, K., Boucheron, E., Vanneste, S., Engler, J.D., Inze, D., and Beeckman, T. (2002). Auxin-mediated cell cycle activation during early lateral root initiation. Plant Cell 14, 2339-2351. DOI: http://dx.doi.org/10.1105/tpc.004960.

Ichihashi, Y., Kawade, K., Usami, T., Horiguchi, G., Takahashi, T., and Tsukaya, H. (2011). Key Proliferative Activity in the Junction between the Leaf Blade and Leaf Petiole of Arabidopsis. Plant Physiology 157, 1151-1162. DOI: http://dx.doi.org/10.1104/pp.111.185066.Keller, C.P., Stahlberg, R., Barkawi, L.S., and Cohen, J.D. (2004). Long-term inhibition by auxin of leaf blade expansion in bean and arabidopsis. Plant Physiology 134, 1217-1226. doi: 10.1104/pp.103.032300.

Lee, S.-W., Feugier, F.G., and Morishita, Y. (2014). Canalization-based vein formation in a growing leaf. J Theor Biol 353, 104-120. . DOI: 10.1016/j.jtbi.2014.03.005.

Mähönen, A.P., ten Tusscher, K., Siligato, R., Smetana, O., Díaz-Triviño, S., Salojärvi, J., Wachsman, G., Prasad, K., Heidstra, R., and Scheres, B. (2014). PLETHORA gradient formation mechanism separates auxin responses. Nature 515, 125-129. DOI: 10.1038/nature13663.

Mashiguchi, K., Tanaka, K., Sakai, T., Sugawara, S., Kawaide, H., Natsume, M., Hanada, A., Yaeno, T., Shirasu, K., Yao, H., et al. (2011). The main auxin biosynthesis pathway in Arabidopsis. Proceedings of the National Academy of Sciences of the United States of America 108, 18512-18517. DOI: 10.1073/pnas.1108434108.

Mattsson, J., Ckurshumova, W., and Berleth, T. (2003). Auxin signaling in Arabidopsis leaf vascular development. Plant Physiology 131, 1327-1339.

Mattsson, J., Sung, Z.R., and Berleth, T. (1999). Responses of plant vascular systems to auxin transport inhibition. Development 126, 2979-2991.

Merks, R.M.H., Guravage, M., Inzé, D., and Beemster, G.T.S. (2011). VirtualLeaf: an open-source framework for cell-based modeling of plant tissue growth and development. Plant Physiol 155, 656666. DOI: $10.1104 /$ pp.110.167619.

Mitchison, G.J. (1980). The Dynamics of Auxin Transport, Vol 209.

Mitchison, G.J. (1981). The Polar Transport of Auxin and Vein Patterns in Plants. Philos T Roy Soc B 295, 461-471. DOI: 10.1098/rstb.1981.0154.

Nishimura, T., Hayashi, K., Suzuki, H., Gyohda, A., Takaoka, C., Sakaguchi, Y., Matsumoto, S., Kasahara, H., Sakai, T., Kato, J., et al. (2014). Yucasin is a potent inhibitor of YUCCA, a key enzyme in auxin biosynthesis. Plant Journal 77, 352-366. DOI: 10.1111/tpj.12399.

Okada, K., Ueda, J., Komaki, M.K., Bell, C.J., and Shimura, Y. (1991). Requirement of the Auxin Polar Transport System in Early Stages of Arabidopsis Floral Bud Formation. The Plant Cell Online 3, 677684. DOI: 10.1105/tpc.3.7.677.

Onoda, Y., Schieving, F., and Anten, N.P.R. (2015). A novel method of measuring leaf epidermis and mesophyll stiffness shows the ubiquitous nature of the sandwich structure of leaf laminas in broadleaved angiosperm species. J Exp Bot 66, 2487-2499. DOI: 10.1093/jxb/erv024.

Pacheco-Villalobos, D., Diaz-Moreno, S.M., van der Schuren, A., Tamaki, T., Kang, Y.H., Gujas, B., Novak, O., Jaspert, N., Li, Z.N., Wolf, S., et al. (2016). The Effects of High Steady State Auxin Levels on Root Cell Elongation in Brachypodium. Plant Cell 28, 1009-1024. DOI: 10.1105/tpc.15.01057.

Perrot-Rechenmann, C. (2010). Cellular Responses to Auxin: Division versus Expansion. Cold Spring Harbor Perspectives in Biology 2. DOI: 10.1101/cshperspect.a001446.

Petersson, S.V., Johansson, A.I., Kowalczyk, M., Makoveychuk, A., Wang, J.Y., Moritz, T., Grebe, M., Benfey, P.N., Sandberg, G., and Ljung, K. (2009). An Auxin Gradient and Maximum in the Arabidopsis Root Apex Shown by High-Resolution Cell-Specific Analysis of IAA Distribution and Synthesis. Plant Cell 21, 1659-1668. DOI: 10.1105/tpc.109.066480. 
Petrasek, J., Mravec, J., Bouchard, R., Blakeslee, J.J., Abas, M., Seifertova, D., Wisniewska, J., Tadele, Z., Kubes, M., Covanova, M., et al. (2006). PIN proteins perform a rate-limiting function in cellular auxin efflux. Science 312, 914-918. DOI: 10.1126/science.1123542. Rolland-Lagan, A.G., and Prusinkiewicz, P. (2005). Reviewing models of auxin canalization in the context of leaf vein pattern formation in Arabidopsis. Plant Journal 44, 854-865. DOI:10.1111/j.1365313X.2005.02581.x. and the eye: a parsimonious theory for scaling and growth control? Wiley Interdiscip Rev Dev Biol. 4, 591-608. DOI: 10.1002/wdev.195. et al. (2008). Rapid synthesis of auxin via a new tryptophan-dependent pathway is required for shade avoidance in plants. Cell 133, 164-176. doi: 10.1016/j.cell.2008.01.049. Development and Morphogenesis. Current Biology 24, 475-R483. DOI: 10.1016/j.cub.2014.03.014. Sawchuk, M.G., Edgar, A., and Scarpella, E. (2013). Patterning of Leaf Vein Networks by Convergent Auxin Transport Pathways. PLoS Genetics 9. DOI: 10.1371/journal.pgen.1003294. Scarpella, E., Francis, P., and Berleth, T. (2004). Stage-specific markers define early steps of procambium development in Arabidopsis leaves and correlate termination of vein formation with mesophyll differentiation. Development 131, 3445-3455. DOI:10.1242/dev.01182. Scarpella, E., Marcos, D., Friml, J., and Berleth, T. (2006). Control of leaf vascular patterning by polar auxin transport. Genes Dev 20, 1015-1027. DOI:10.1101/gad.1402406.

Scheres, B., and $\mathrm{Xu}, \mathrm{L}$. (2006). Polar auxin transport and patterning: grow with the flow. Genes \& Development 20, 922-926. DOI:10.1101/gad.1426606. Sieburth, L.E. (1999). Auxin is required for leaf vein pattern in Arabidopsis. Plant Physiology 121, 11791190. DOI: http://dx.doi.org/10.1104/pp.121.4.1179.

Stepanova, A.N., Robertson-Hoyt, J., Yun, J., Benavente, L.M., Xie, D.Y., Dolezal, K., Schlereth, A., Jurgens, G., and Alonso, J.M. (2008). TAA1-mediated auxin biosynthesis is essential for hormone crosstalk and plant development. Cell 133, 177-191. DOI: 10.1016/j.cell.2008.01.047.

Stepanova, A.N., Yun, J., Robles, L.M., Novak, O., He, W., Guo, H., Ljung, K., and Alonso, J.M. (2011). The Arabidopsis YUCCA1 Flavin Monooxygenase Functions in the Indole-3-Pyruvic Acid Branch of Auxin Biosynthesis. Plant Cell 23, 3961-3973. DOI: 10.1105/tpc.111.088047.

Thomson, K.S., Hertel, R., Muller, S., and Tavares, J.E. (1973). 1-N-Naphthylphthalamic Acid and 2,3,5Triiodobenzoic Acid - in-Vitro Binding to Particulate Cell Fractions and Action on Auxin Transport in Corn Coleoptiles. Planta 109, 337-352. DOI: 10.1007/BF00387102.

Tsugeki, R., Ditengou, F.A., Sumi, Y., Teale, W., Palme, K., and Okada, K. (2009). NO VEIN Mediates Auxin-Dependent Specification and Patterning in the Arabidopsis Embryo, Shoot, and Root. Plant Cell 21, 3133-3151. DOI: 10.1105/tpc.109.068841.

Wartlick, O., Mumcu, P., Julicher, F., and Gonzalez-Gaitan, M. (2011). Understanding morphogenetic growth control - lessons from flies. Nat Rev Mol Cell Bio 12, 594-604. doi: 10.1038/nrm3169.

Won, C., Shen, X., Mashiguchi, K., Zheng, Z., Dai, X., Cheng, Y., Kasahara, H., Kamiya, Y., Chory, J., and Zhao, Y. (2011). Conversion of tryptophan to indole-3-acetic acid by TRYPTOPHAN AMINOTRANSFERASES OF ARABIDOPSIS and YUCCAs in Arabidopsis. Proceedings of the National Academy of Sciences of the United States of America 108, 18518-18523. DOI: 10.1073/pnas. 1108436108

Zhao, Y. (2012). Auxin biosynthesis: a simple two-step pathway converts tryptophan to indole-3-acetic acid in plants. Mol Plant 5, 334-338. . DOI: 10.1093/mp/ssr104. 
504 Figure 1. Auxin biosynthesis mutants treated with auxin. (A) Vein patterning in 12 day-old leaves

505 WT (Col-0) and auxin biosynthesis mutant wei8-1 tar2-1 grown in presence or absence of $1 \mu \mathrm{M}$ IAA.

506 (B) Quantification of leaf surface area of plants presented in (A). Asterisk ( $\left.{ }^{\star \star \star}\right)$ indicates significant

507 difference at $\mathrm{P}<0.001$ (T-test). Data are mean ( $n=40 \pm$ s.e.). n.s., non-significant difference at $\mathrm{P}<0.05$

508 (T-test). Scale Bar, $1 \mathrm{~mm}$.

509

510 Figure 2. Time resolved auxin biosynthesis map in Arabidopsis leaf. Combinatorial expression 511 of auxin biosynthetic genes (TAA1, TAR2, YUC1, YUC2, YUC4) at 2 DAG (C-H), 3 DAG (I-M) and 4 DAG

512 (N-Q). (A) Longitudinal (above) and transverse (below) leaf sections. The blue dashed line defines the 513 separation between adaxial (ad) and abaxial (ab) leaf polarity. (B) Schematic representation of the 514 major auxin biosynthesis pathway (Trp, tryptophan; IPA, indole 3-pyruvic acid; IAA, indole3-acetic 515 acid). (C-Q) Time resolved expression pattern of pTAA1::TAA1-GFP (C, D, I, J, N), TAR2::GUS (E, L, P),

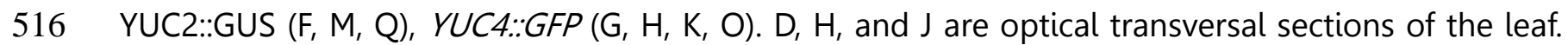

517 Asterisks: yellow asterisks indicate TAA1 and YUC4 expression in the epidermis; white asterisks 518 indicate TAA1 and YUC4 expression in the lamina; black asterisks show TAR2 and YUC2 expression in 519 provascular tissues. SAM indicates the position of the shoot apical meristem. Scale bar, $20 \mu \mathrm{m}$ unless

520 otherwise indicated. DAG, days after germination. The data supporting auxin biosynthesis in 5 DAG 521 leaf are presented in Figures S1 and S2.

523 Figure 3. Effect of NPA on leaf vein patterning, auxin biosynthesis and distribution. (A) Vein

524 patterning in 12 day-old leaves grown in presence of increasing concentrations of NPA. (B-F), 525 Immunodetection of both TAA1-GFP and PIN1 in 4 day-old leaves grown in presence of NPA. Boxed 526 Inset in (D) shows polar PIN1 at the plasma membrane of vascular cells arrowed in (D) and (E). F, 527 Transversal sections of leaves presented in B and C, respectively. White dashed lines indicate leaf 
528 boundaries. Yellow dashed lines indicate pre-provascular cells expressing both TAA1 (red) and PIN1

529 (green). Note the proliferation of pre-provascular cells in a concave shape in $10 \mu \mathrm{M}$ treated leaf. G,

530 Percentage of cells expressing both PIN1 and TAA1 in leaf lamina. By dividing by the total number of

531 cells constituting leaf primordium. Stars $\left(^{*}\right)$ indicate significant difference to control at $\mathrm{P}<0.01$ (t-test).

532 Data are means ( $n=40 \pm$ s.e.). H, DR5::Venus (auxin levels) and PIN1p::PIN1-GFP in 3, 4 and 5 day-old

533 leaves. Top panel: control leaves. Lower panel: leaves treated with $10 \mu \mathrm{M}$ NPA. Scale, $500 \mu \mathrm{m}$ in (A),

$534200 \mu \mathrm{m}(\mathrm{F})$ and $10 \mu \mathrm{m}(\mathrm{H})$.

535

536 Figure 4. In silico modeling of spontaneous patterning of vascular tissue in leaf primordium.

537 (A) Left, leaf template resembling a leaf primordium. The model assumes local auxin synthesis (dark

538 green colored cells), leaf petiole cells (grey colored cells), a relatively stiff outermost surface of the

539 epidermal layer, non-directional transport of auxin and auxin-dependent cell growth; right TAR2-

540 driven GUS expression in 2-day-old control leaf-primordia. (B) Left, simulation result of our model

541 with the parameter values as given in Table S1. The model was able to reproduce a realistic mid-vein

542 made up of elongated cells and branched vasculature, as observed in 4-day-old control leaf-

543 primordia (see C). Forces on the vertices of a cell in the leaf tissue is shown by an arrow representing

544 the force vector, in the case of a control-leaf; Magenta colored arrows represent forces on the vertices

545 of midvein cells, and blue arrows represent forces on the vertices of other cells in the leaf primordia.

546 Inset shows cellular auxin concentration in the leaf primordia. (C) TAR2-driven GUS expression in a

547 4-day-old control leaf-primordia. (D) Simulation result with a 25-fold lowered rate of auxin transport.

548 All the other parameter values were kept the same as in (C). Inset shows cellular auxin concentration

549 in the leaf primordia. (E) TAR2-driven GUS expression in 3-day-old NPA-treated leaf-primordia. (F)

550 Simulation result for reduced auxin production and reduced transport rate. A realistic midvein could

551 be produced in simulation only when the overall cell area growth rate was decreased, consistent with

552 NPA treatment rescue of an auxin biosynthesis mutant. (G) In silico modeling of PIN1 based auxin 
553 transport. The model incorporates PIN1 based transport of auxin, and is able to reproduce a realistic

554 mid-vein even in the case of no diffusion $(d=0)$ and PIN1 based transport only. Inset shows cellular

555 auxin concentration in the leaf primordia. The arrows indicate the net direction, but not the

556 magnitude of the auxin flux. The lack of clear polarity of the auxin flow in the epidermal cells is likely

557 due to the absence of any external source of epidermal auxin. Asterisks ${ }^{*}$ ) denote provascular cells.

559 Figure 5. Compensation of vein patterning in auxin biosynthesis mutants by NPA. (A) Vein

560 patterning in 10-day old wild-type (WT) leaf. (B) WT leaf after NPA treatment. (C) wei8-1tar2-1 leaf

561 treated with (right) or without (left) $10 \mu \mathrm{M}$ NPA. Leaves were classified according to their vein

562 architecture. Class I, indistinguishable from untreated WT. Leaves contain a single midvein reaching

563 distally to the tip of the leaf. Class II, fused midveins and Class III, fused midveins and increased

564 frequency of fused high order veins. (D) Genotype dependent distribution of Class I, II and III leaves

565 in seedlings treated with NPA. Scale bar, $500 \mu \mathrm{m}$.

567 Figure 6. Auxin does not regulate PIN1 polarisation nor its own biosynthesis. (A), (B) and (C)

568 Immunocytodetection of PIN1 in WT (A), in wei8-1 tar2-1 (B) and in yuc1 yuc4 double mutants (C).

569 Arrowheads indicate PIN1 polarity; dashed circle, intervascular pre-provascular cells expressing PIN1

570 visible in WT but absent in wei8-1 tar2-1 and in yuc1 yuc4 double mutants. (D), Quantification of

571 PIN1, TAA1, TAR1, TAR2, YUC1, YUC2, YUC4 mRNA in 3 day-old WT seedlings incubated in the

572 presence of $1 \mu \mathrm{M}$ or $5 \mu \mathrm{M}$ IAA for 4 hours. (Parentheses) Fraction of primordia showing the displayed

573 features. Scale bar, $20 \mu \mathrm{m}$.

575 Figure 7. Gradual subcellular localization of PIN1 in dynamic TAA1 expression domains. PIN1

576 and TAA1-GFP subcellular localization in 6-day old leaf. (A-C), Dynamic expression pattern of TAA1-

577 GFP and subcellular localization of PIN1 in pre-provascular, provascular and mature vascular cells. 
578 (A), PIN1 localization. (B), TAA1-GFP. (C), Panel (A) and panel (B) merged. (D-F), Higher magnification

579 of images in dotted frames in (A-C), respectively. (G-I), Higher magnification of images in solid frames

580 in (A-C), respectively). Arrowheads indicate PIN1 polarity in provascular cells. Asterisks (*): white

581 asterisks indicate developing provascular tissues, yellow asterisks indicate pre-provascular cells with

582 PIN1 non-polar. Yellow inset in (C) shows respectively polar PIN1 and TAA1 down regulation in

583 mature vasculature. Scale bars, $20 \mu \mathrm{m}(\mathrm{A}-\mathrm{C}), 10 \mu \mathrm{m}(\mathrm{D}-\mathrm{I})$.

585 Methods

586 Plant material and growth conditions. Columbia Arabidopsis ecotype (Col-0) as wild type (WT),

587 YUCp::GUS reporter lines and yuc mutants single and multiple combinations were as previously 588 described (Cheng et al., 2006; Ditengou et al., 2008). Details of pTAA1::TAA1-GFP, wei8-1, wei8-1tar2-

5891 and wei8-1tar1-1tar2-1 and pin1 mutants are as previously described (Stepanova et al., 2008);

590 (Galweiler et al., 1998). Wei8 (Stepanova et al. Cell 2008) is also known as sav3 (Tao et al., 2008). To

591 more accurately reflect the corresponding protein's function, WE/8 was subsequently renamed TAA1

592 TRYPTOPHAN AMINOTRANSFERASE OF ARABIDOPSIS1 by Tao et al. (2008). YUC4p::GFP seeds were

593 obtained from Yunde Zhao. Seeds were surface-sterilized and sown on solid Arabidopsis medium (2.3

$594 \mathrm{~g} /$ liter MS salts, 1\% sucrose, 1.6\% agar-agar ( $\mathrm{pH}$ 6.0) adjusted with $\mathrm{KOH}$ ). After vernalization for 2 days

595 at $4^{\circ} \mathrm{C}$, seeds were germinated under a long day period (16h light, $8 \mathrm{~h}$ darkness). In polar auxin

596 transport inhibitory experiments, seedlings were grown on media supplemented with $10 \mu \mathrm{M}$ NPA for

5970 to 10 days.

598

599 Immunocytodetection. Seedlings of different ages were fixed with 4\% paraformaldehyde in PBS ( $\mathrm{pH}$

600 7.3) and used for whole-mount in situ immunolocalisation as previously described (Ditengou et al.,

601 2008). PIN1 was detected using a mouse anti-PIN1 monoclonal antibody (1:100) and TAA1-GFP with 602 a rabbit anti-GFP antibody (1:600) (Molecular Probes). YUC4p::GFP was detected with a rabbit anti-GFP 
603 polyclonal antibody (1:200). Samples were incubated with secondary antibodies (Alexa 488 goat anti 604 mouse and Alexa 555 goat anti rabbit from Invitrogen, both at 1:1000 dilution).

606 Microscopy and analysis. Histological detection of - -glucuronidase (GUS) activity was performed 607 according to (Scarpella et al., 2004). For analysis of vascular patterns, seedlings were cleared in $100 \%$ 608 ethanol overnight. They were re-hydrated and dissected under 50\% glycerol, then mounted in chloral 609 hydrate:glycerol:water (8:3:1, w/v/v). GFP plants were fixed with 4\% formaldehyde at room temperature 610 and mounted in Prolong Gold antifade reagent (Molecular Probes). For light microscopy, samples were 611 observed with a Zeiss Axiovert 200M MOT (Carl Zeiss, Goettingen, Germany) for high magnification

612 pictures. By contrast, low magnification views were taken with a Zeiss Stemi SV11 Apo 613 stereomicroscope (Carl Zeiss, Goettingen, Germany), viewed under differential interference contrast 614 (DIC) optics or dark field illumination. Fluorescent proteins were analyzed with a Zeiss LSM 5 DUO 615 scanning microscope or AZ-C1 Macro Laser Confocal Microscope (NIKON GMBH, Düsseldorf, 616 Germany). GFP was excited using the 488nm laser line in conjunction with a 505-530 band-pass filter.

617 To simultaneously monitor Alexa488 and Alexa 555 fluorescences, we used multi-tracking in frame 618 mode and the emission was separated using the on-line unmixing feature of the Meta spectral 619 analyzer. Images were extracted and analyzed with the Zen2009 software (Carl Zeiss Microlmaging) 620 and representative of at least 20 individual plants.

622 Real time RT-PCR. The effect of auxin on gene expression was quantified by real-time quantitative 623 RT-PCR. Arabidopsis seeds were deposited on 6-7 mm filter paper strips lying at the surface of solid 624 Arabidopsis growth medium (see above). This procedure facilitates the transfer of seedlings on the 625 filter paper from Agar to liquid Arabidopsis growth medium (not containing Agar) supplemented with 626 or without IAA. Three-day old Arabidopsis seedlings were transferred to medium containing either 627 mock (control) or IAA. After treatment the filter paper strips with seedlings were transferred to RNA- 
628 later solution (Ambion). Total RNA was extracted using the RNeasy Micro Kit (Qiagen). Reverse

629 transcription was performed using $1 \mu \mathrm{g}$ of total RNA and RevertAid M-MulV reverse transcriptase

630 (Fermentas, St Leon-Rot, Germany) according to the manufacturer's instructions. qRT-PCR was

631 performed using Maxima SYBR Green kit (Fermentas) on a Light cycler480 Real-Time system (Roche,

632 Mannheim, Germany). The gene-specific primers for qRT-PCR are listed in Table S2. The efficiency of

633 each primer pair was determined by examination of a standard curve using serial dilutions of genomic

634 DNA. The PCR was performed using a three-step protocol including melting curve analysis. The

635 relative gene expression was analyzed using the $\Delta-\Delta$ cycle threshold method. ACTIN2 served reference

636 gene, three biological replicates and three technical replicates were used to evaluate gene expression.

638 Image post-processing. All images depicting PIN1/TAA1 double labeling or PIN1/DAPI are 3D 639 reconstructions of optical sections with Imaris 7.4.0 (Bitplane AG, Zurich, Switzerland). All images were 640 assembled using Microsoft PowerPoint 2013.

\section{Computational model description.}

643 A cell-based modeling framework, Virtual Leaf, that couples vertex dynamics and chemical dynamics, 644 was adapted to study the vein patterning in leaves in silico (Merks et al., 2011). We simplified the 645 model system by representing the longitudinal section of leaf primordia by a two-dimensional 646 network of interconnected polygons (cells), specified by the surrounding vertices. Each cell, in this 647 framework, is characterized by an energy function which describes the balance between turgor 648 pressure and cell wall stiffness,

$$
E=\lambda_{A} \sum_{\alpha}\left(A_{\alpha}-A_{\alpha}^{0}\right)^{2}+\lambda_{s} \sum_{<i j>}\left(l_{i j}-l_{i j}^{0}\right)^{2}
$$

$650 A_{\alpha}$ is the area of the cell $\alpha$ and $A_{\alpha}^{0}$ is the preferred (rest) area of that cell. $l_{i j}$ is the length of the cell 651 wall linking nodes $i$ and $j$ of the polygons and the sum over $\langle i j\rangle$ is over all links. $\lambda_{A}$ is a parameter 
652 setting the cell resistance to compression or expansion and $\lambda_{s}$ describes the cell wall stiffness. Cell

653 behaviors, like expansion, division and active shape changes are described by minimization of the

654 energy function $E$. The minimization depends on the dimensionless ratio $\lambda_{A} l_{0}^{2} / \lambda_{s}$, where $l_{0}$ is a cell

655 size dependent lengthscale. The value of the dimensionless ratio used in our simulations was 0.01 , in

656 accordance with (Merks et al., 2011).

657 To study vein patterning in developing leaf primordia, we created a leaf template to resemble the

658 tissue of a leaf primordium, as in Fig. 5A. Cells at the base of this template (cells colored in gray in Fig.

$6595 \mathrm{~A}$ ) were assigned with very high cell stiffness $\lambda_{s}$, in order to implement proper boundary conditions,

660 namely the effect of the firm attachment of the leaf primordia to the shoot apical meristem. The

661 outermost layer of cells in the leaf primordia represents the stiff epidermal layer. To incorporate these

662 properties of the epidermal cells in our model, the outermost cell walls of the cells at the perimeter of

663 the leaf primordium were assumed to have higher stiffness than the internal cell walls. We used a value

664 for the ratio of perimeter to internal cell wall stiffness within the experimentally reported range, i.e

$665 \lambda_{s}$ (perimeter) $=12 \lambda_{s}$ (interior) (Gibson et al., 1988; Onoda et al., 2015). In our model auxin is

666 synthesized locally in only few cells and subsequently auxin diffuses across the neighboring cells in

667 the tissue. The leaf primordia in our model consists of three different cell types:

668 (1) auxin synthesizing cells (colored in dark green in Fig. 4A),

669 (2) cells that do not synthesize auxin (colored in light green in Fig. 4A),

670 (3) petiole cells (colored in grey in Fig. 4A).

671 The latter is a computational cell type aimed to simulate the firm attachment of the leaf to the

672 meristem and the drainage of auxin.

673 The general dynamic equation governing the amount of auxin $n_{\alpha}$ in a cell is given by:

$$
\frac{\partial n_{\alpha}}{\partial t}=s(\alpha)+d \sum_{\beta} l^{\alpha \beta}\left(\frac{n_{\beta}}{A_{\beta}}-\frac{n_{\alpha}}{A_{\alpha}}\right)
$$


675 Here $l^{\alpha \beta}$ is the length of the cell wall separating cells $\alpha$ and $\beta$, across which the auxin diffusion takes

676 place and $A_{\alpha}$ is the area of the cell $\alpha$. The first term describes auxin production at a constant rate

$677 s(\alpha)=s_{0} \delta_{\alpha, p}$, non-zero only for the auxin producer cells $p$. Auxin production is limited to only few

678 cells (cell colored in dark green in Fig. 4A). The second term accounts for auxin spreading in the tissue

679 of leaf primordia by a diffusion process, where $d$ is a constant that measures the speed of inter-cellular

680 auxin transport and is related to the auxin diffusion coefficient. Auxin is drained through cells located

681 at the base of leaf primordia. To simulate this auxin drainage, from these cells (termed "petiole cells"

682 and colored in grey in Fig. 4A), we implement perfectly absorbing boundary condition at the petiole

683 cell walls.

684 The typical cell size in the simulation has a length of about $l_{0} \cong 10 \mu \mathrm{m}$. We used the experimentally

685 reported range, $10^{-5}-10^{-8} \mathrm{~ms}^{-1}$, for the value of speed of auxin transport $d$ (Mitchison, 1980).

686 At time $t=0$ the amount of auxin is $n=0$ in all the cells in the leaf primordia. For $t>0$ auxin is

687 produced only in auxin synthesizing cells at a constant rate $s_{0}$. Motivated by experimental images of

688 a 2DAG leaf, in our simulation we start initially with only four auxin synthesizing cells, but our

689 qualitative results are not dependent on the initial geometry (Fig. 4A left and right panel and Fig. S7).

690 All the cells in the leaf primordia grow by increasing their target area at the same constant rate $g_{0}=$

$691610^{-14} \mathrm{~m}^{2} \mathrm{~s}^{-1}$ and divide over their shortest axis once their area is doubled. However, in any cell except

692 the auxin synthesizing cell, if the auxin concentration increases beyond a threshold value $c^{*}=$ $6932.410^{9} \mathrm{~m}^{-2}$, then the growth rate of that cell increases to a value $g>g_{0}$. We tested a broad range of 694 cell area growth rates $g$ and found that main vein formation and main vein bifurcation is a robust

695 feature appearing for the values of ratio $g / g_{0} \gtrsim 3$ and up to at least several hundreds (Fig. S8).

696 The force $F\left(X_{i}\right)$ on each node $i$ at vector position $X_{i}$ of a cell is given by:

$697 \quad F\left(X_{i}\right)=-\frac{\partial E}{\partial X_{i}}(3)$

$698=-\lambda_{A} \sum_{\alpha, i \in \alpha}\left(A_{\alpha}-A_{\alpha}^{0}\right)\left[R\left(X_{i+1}^{\alpha}-X_{i-1}^{\alpha}\right)\right]+\lambda_{s} \sum_{j}\left(l_{i j}-l_{i j}^{0}\right) \tilde{\mathbf{u}}_{X_{i} X_{j}}(4)$ 
where $R$ is the 90 degree rotation matrix:

700

$$
R=\left[\begin{array}{cc}
0 & -1 \\
1 & 0
\end{array}\right]
$$

$701 \tilde{\mathbf{u}}_{X_{i} X_{j}}$ is the unit vector joining the vertices at position $X_{i}$ and $X_{j}$, the summation $\sum_{\alpha, i \in a}$ is over all 702 cells that contain node $i$ and $X_{i+1}^{\alpha}\left(X_{i-1}^{\alpha}\right)$ denotes the subsequent (antedecent) to node $i$ as the nodes

703 in cell $\alpha$ are traversed clockwise.

704 The force vector field of an in silico growing leaf primordium is shown by arrows in Fig. 4B,D,F. Magenta

705 colored arrows represent net force acting on the vertices of auxin producing cells, whereas blue

706 colored arrows represent net force acting on the vertices of non-auxin producing cells in the leaf

707 primordia. The values for the model parameters are shown in Table S1.

708

PIN1 based auxin transport. In addition to the passive diffusive transport of auxin, we also studied,

710 in our model, the effect of active transport of auxin via PIN1 only. The general dynamic equation

711 governing the amount of auxin $n_{\alpha}$ in cell $\alpha$ in this case is given by:

712

713

$$
\frac{\partial n_{\alpha}}{\partial t}=s_{0}(\alpha)+\tau \sum_{\beta}\left(P_{\beta \mid \alpha} \frac{n_{\beta}}{A_{\beta}}-P_{\alpha \mid \beta} \frac{n_{\alpha}}{A_{\alpha}}\right)
$$

714 Here $P_{\beta \mid \alpha}$ is the amount of PIN1 on the cell wall of cell $\beta$ neighboring cell $\alpha, \tau$ is the constant related

to the auxin transport via PIN1 and $A_{\beta}$ is the area of cell $\beta$. The above equation is an extension of

716 Equation (1) given above with $d=0$. The only addition being the second term on the right hand side

717 of Equation S1, which describes the intercellular transport of auxin via PINL.

718 The rate of attachment of PIN1 to a given cell wall that separates cell $\alpha$ and cell $\beta$ depends on the

719 amount of auxin in that particular cell, $\alpha$ and its neighboring cell $\beta$, as well as the amount of

720 cytoplasmic PIN1, $P_{\alpha}$ in that cell. The flux of PIN1 molecules attaching to a given cell wall is given by:

$$
\begin{gathered}
\varphi_{\alpha \mid \beta}=\left(n_{\alpha}-n_{\beta}\right), \quad \text { if } n_{\alpha}>n_{\beta} \\
=0, \quad \text { if } n_{\alpha} \leq n_{\beta}
\end{gathered}
$$


724 Equation governing the amount of PIN1 on the wall of a given cell is given by:

$$
\frac{d P_{\alpha \mid \beta}}{d t}=k_{o n} \varphi_{\alpha \mid \beta} P_{\alpha}
$$

726 Here, $P_{\alpha}$ is the amount of cytoplasmic PIN1 in cell $\alpha$ and $k_{o n}$ is the rate of association of cytoplasmic

727 PIN1 to the wall of cell $\alpha$ neighboring cell $\beta$.

728 The dynamic equation governing the amount of cytoplasmic PIN1, $P_{\alpha}$ in cell $\alpha$ is given by:

$$
\frac{d P_{\alpha}}{d t}=-\sum_{\beta} k_{o n} \varphi_{\alpha \mid \beta} P_{\alpha}+p_{0} .(S 3)
$$

where $p_{0}$ is the rate of PIN1 production in each and every cell.

732 Figure $6 \mathrm{G}$ shows the simulation result of our model including the PIN1 based auxin transport,

733 described by Equations S1-S3, with the parameter values given in Table S3.

734 Our simulation results show the formation of a normal mid-vein also in the case of no diffusion $(d=$

735 0) and PIN1 only transport, see Figure 4G (main text).

Compensation of vein patterning in auxin biosynthesis mutants by NPA. In our model, the area

growth rate of non-auxin producing cells depend on the cellular auxin concentration. When the auxin

739 concentration in a non-auxin producing cell crosses a specified threshold, its area growth rate

740 increases, as a result these cells grow faster than the auxin producing cells, inducing transverse forces,

741 acting laterally on the walls of midvein cells, much larger in magnitude than the forces acting on other

742 cells in the leaf primordia. This prevents the midvein cells from proliferating, thus resulting in a thin

743 vascular strand of elongated cells.

744 In this section, using our model, we will see how reducing auxin production rate requires a

745 simultaneous reduction in auxin transport rate as well as overall cell area growth rate in order to 746 recover wild-type midvein patterning. Our model therefore proposes a mechanism through which a 
747 reduction in auxin biosynthesis can rescue the NPA-induced defects caused by auxin accumulation in

748 the midvein.

749 Continuous auxin production in auxin synthesizing cells and inter-cellular auxin transport sets up an

750 auxin concentration gradient in the growing leaf primordia. Therefore, auxin concentration threshold,

751 beyond which non-auxin producing cells start to grow faster, depends upon several kinetic parameters

752 that also determine auxin concentration gradient.

753 Auxin concentration of a cell in a growing leaf lamina changes according to the following equation:

$$
\frac{\partial c_{\alpha}(x, t)}{\partial t}=s(\alpha)+D \frac{\partial^{2} c_{\alpha}(x, t)}{\partial x^{2}}(S 4)
$$

755 where, $c_{\alpha}(x, t)$ is the auxin concentration of cell $\alpha$ at position $x$ and time $t$ in the leaf primordia,

$756 s(\alpha)=s_{0} \delta_{\alpha, p}$, describes auxin production at a constant rate $s_{0}$, non-zero only for auxin producing cells

$757 p$, and $D$ is the auxin diffusion. Since leaf primordia is growing in area due to an irreversible growth in

758 the area of an individual cell, as a result, the cellular auxin concentration $c=n / A$, where $n$ is the

759 number of auxin molecules in a cell of area $A$, is effected,

$$
\frac{\partial c_{\alpha}(x, t)}{\partial t}=\frac{\frac{\partial n_{\alpha}}{\partial t}}{A_{\alpha}}-\frac{n_{\alpha}}{A_{\alpha}{ }^{2}} \frac{\partial A_{\alpha}}{\partial t}
$$

762 The first term in Equation (S4) describes the change in auxin concentration due to auxin transport, and

763 is given by Equation (2) in the main text. The second term describes the change in auxin concentration

764 as result of dilution of auxin due to an increase in cell area due to cell area growth. Similar situation

765 of an area growth induced dilution of a chemical specie, growth hormone, morphogens, etc., is

766 encountered in other biological systems (Romanova-Michaelides et al., 2015; Wartlick et al., 2011).

768 The change in auxin concentration of cell $\alpha$ at position $x$ and time $t$, can thus be written down as:

$$
\frac{\partial c_{\alpha}(x, t)}{\partial t}=s(\alpha)+D \frac{\partial^{2} c_{\alpha}}{\partial x^{2}}-g c_{\alpha}(S 5)
$$


770 where, $g$ is the area growth of a cell. The steady state solution of Equation (S5) for a delta source of

771 auxin is given by:

772

$$
c=\frac{s_{0}}{\sqrt{g D}\left(1-e^{-2 \lambda L}\right)}\left(e^{-2 \lambda L} e^{\lambda x}+e^{-\lambda x}\right) \text { for } x \geq 0 .(S 6)
$$

773

774 Equation (S6) shows the dependence of auxin concentration threshold $c^{*}$ on auxin production rate $s_{0}$

775 auxin diffusion rate $D$, and cell area growth rate $g$. Auxin diffusion rate is related to the auxin

776 transport rate $d$, which is the actual parameter used in our model to describe auxin transport. It can

777 be seen that lowering auxin production $s_{0}$, as in auxin biosynthesis mutants, requires a simultaneous

778 lowering of auxin transport rate $d$, similar to NPA treatment, as well as overall cell area growth rate $g$

779 (Figures S7 and 4F, main text). 

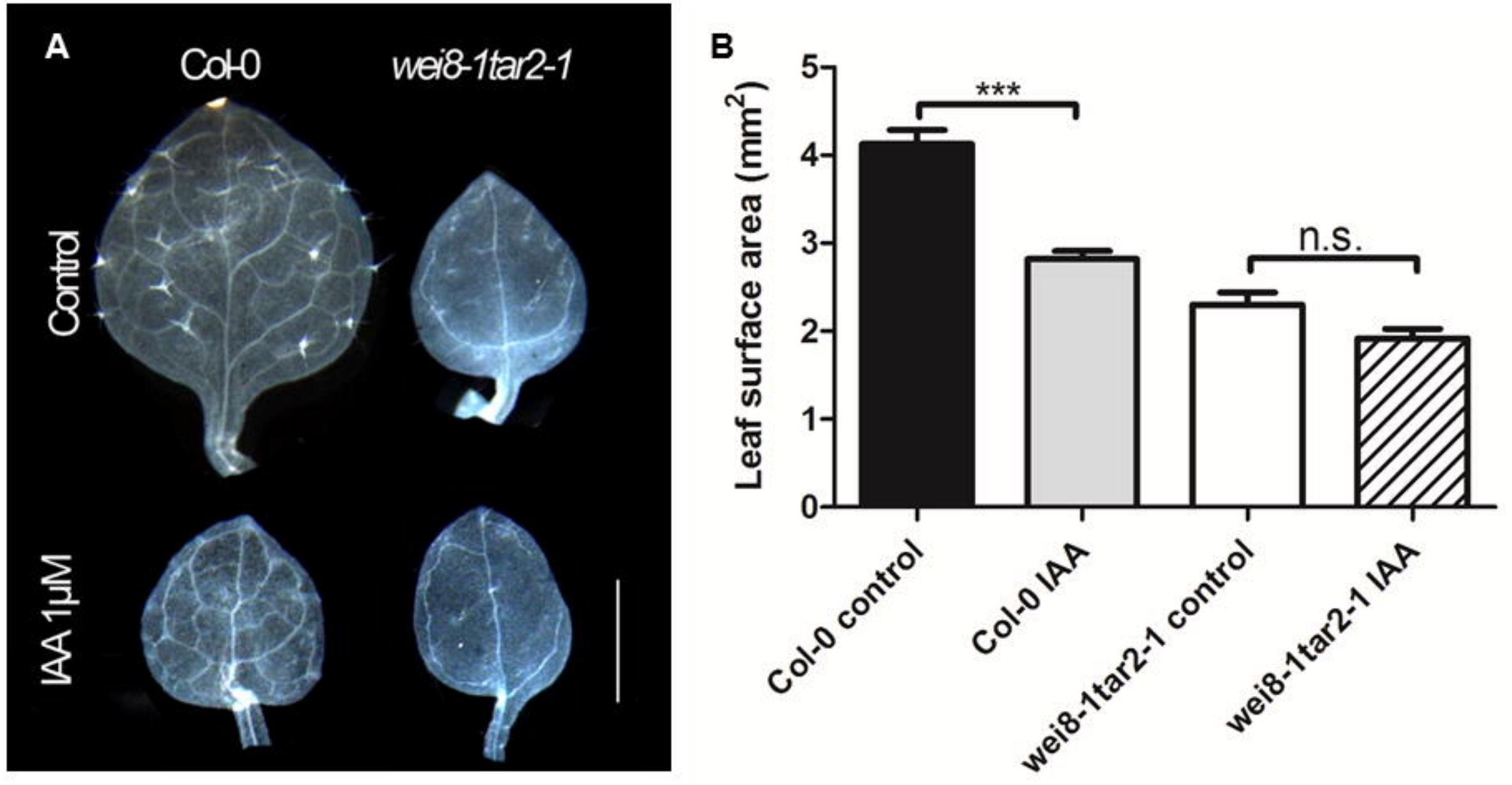

Fig. 1 

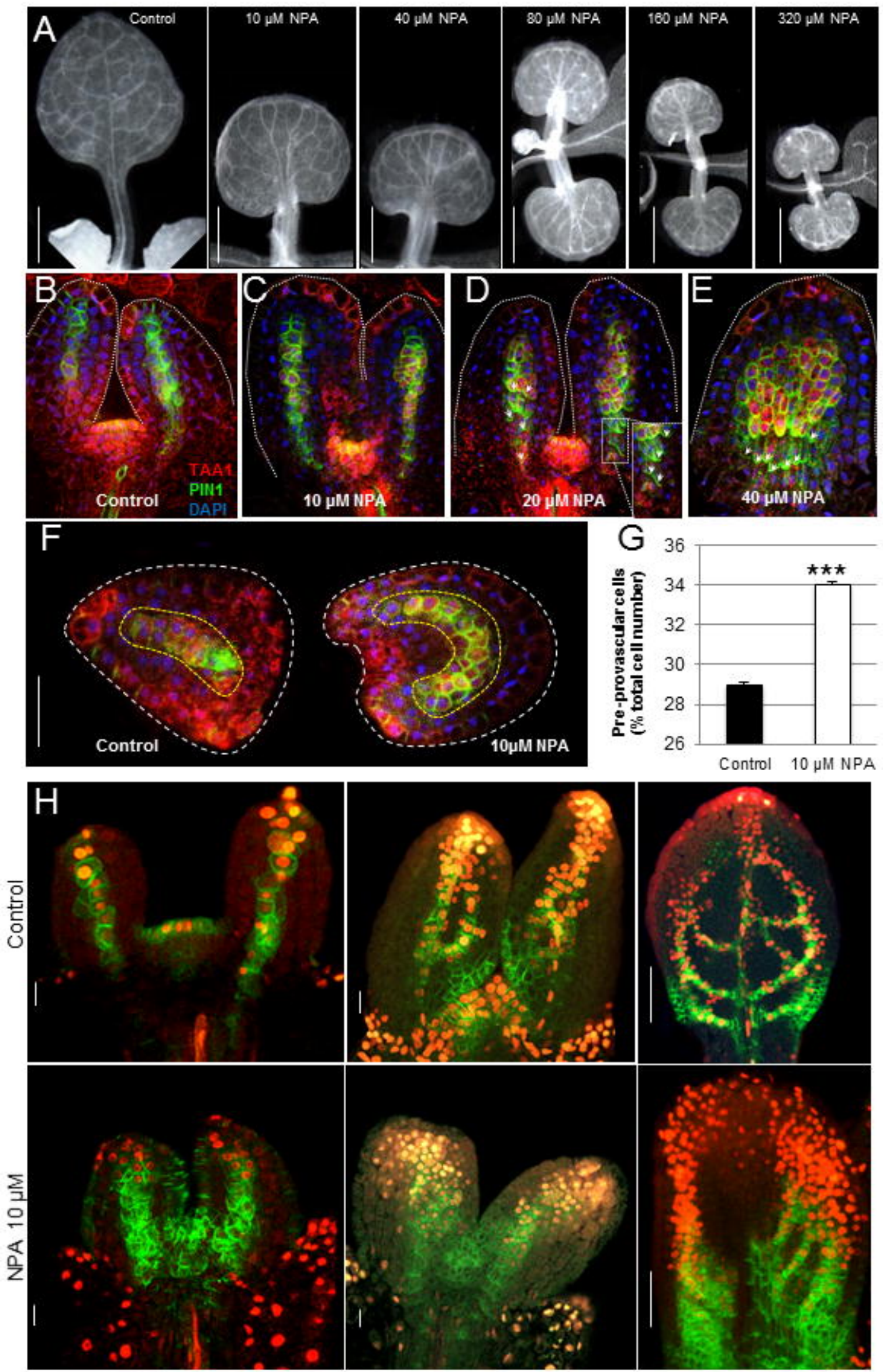


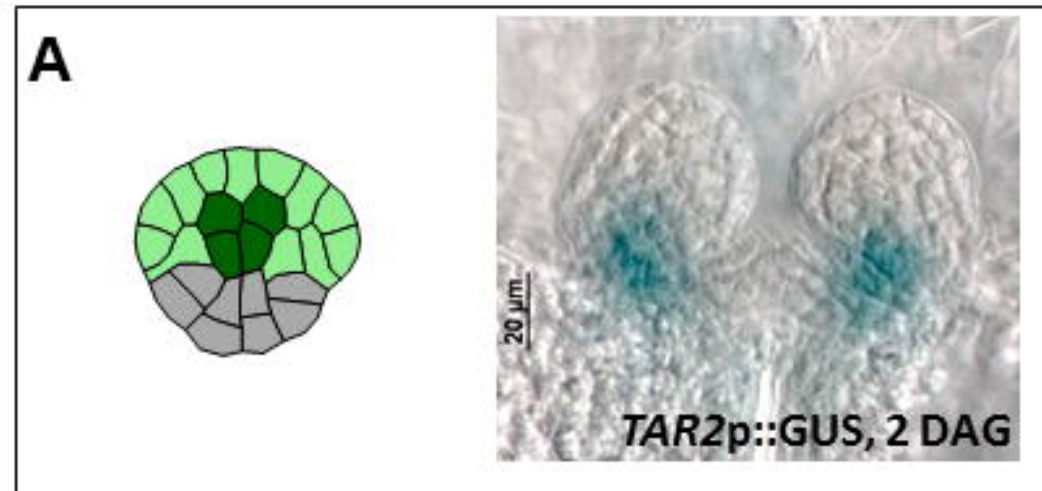

auxin producer cell

petiole cell

low high auxin concentration
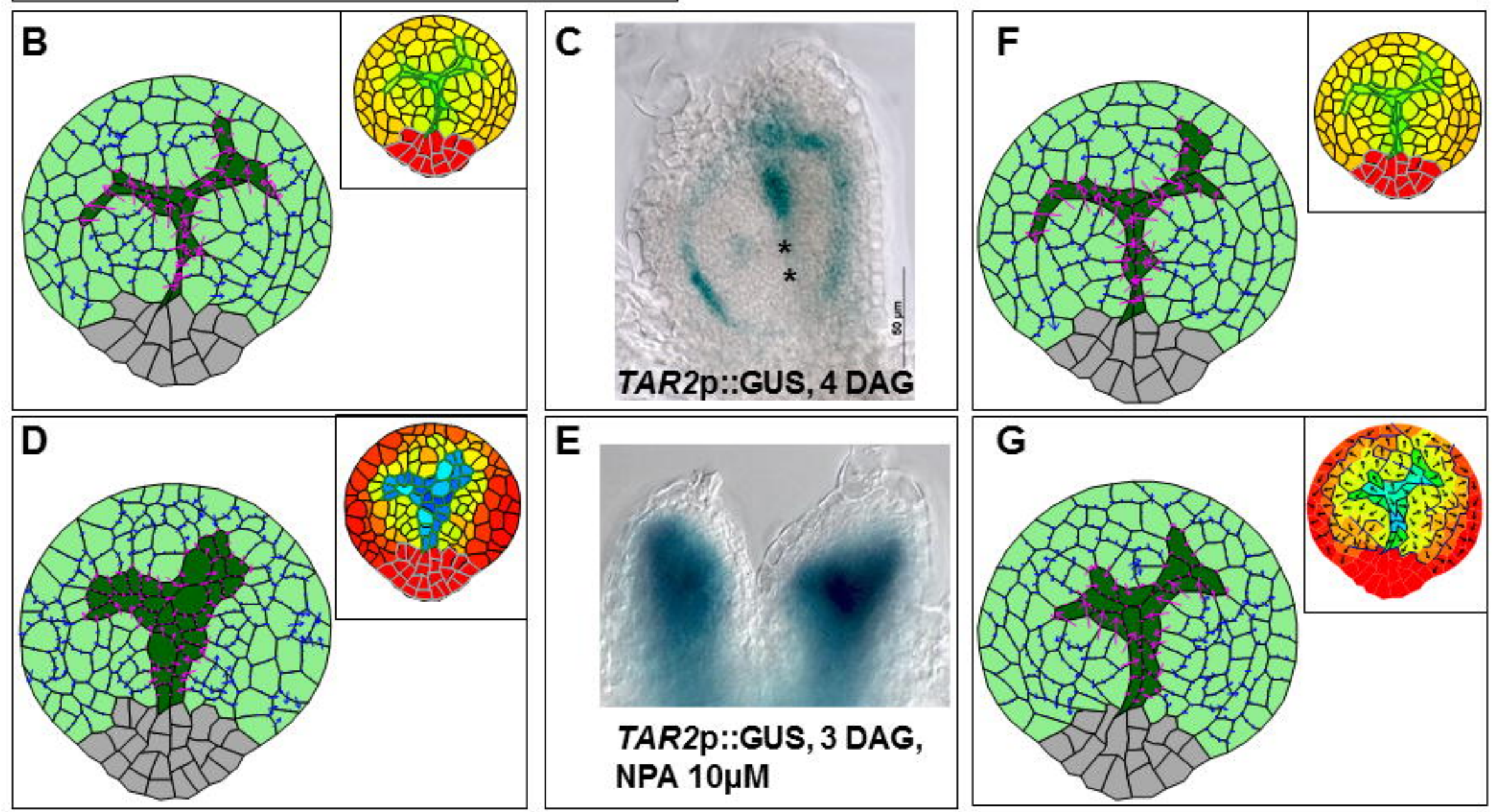

Fig. 4 


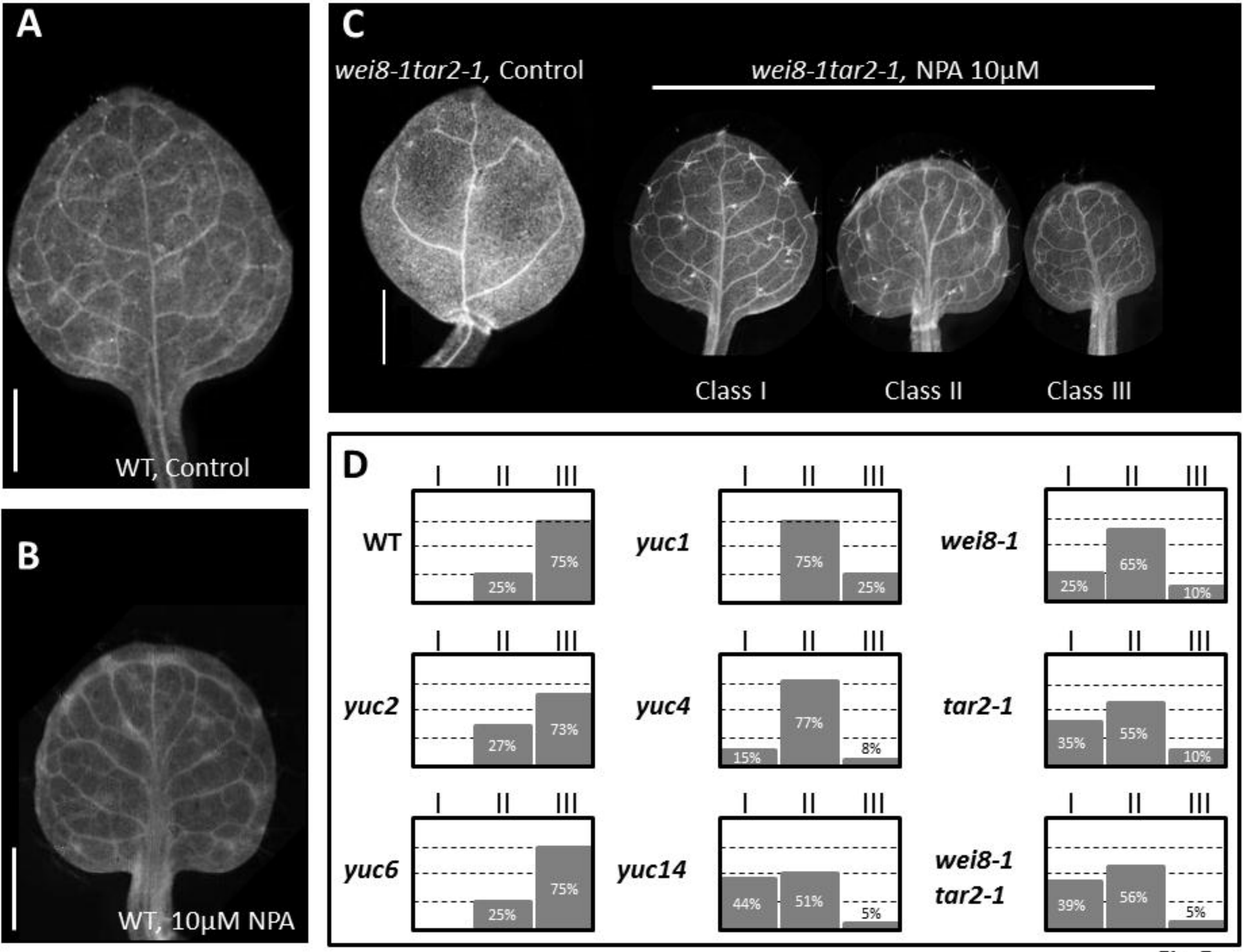

Fig. 5 

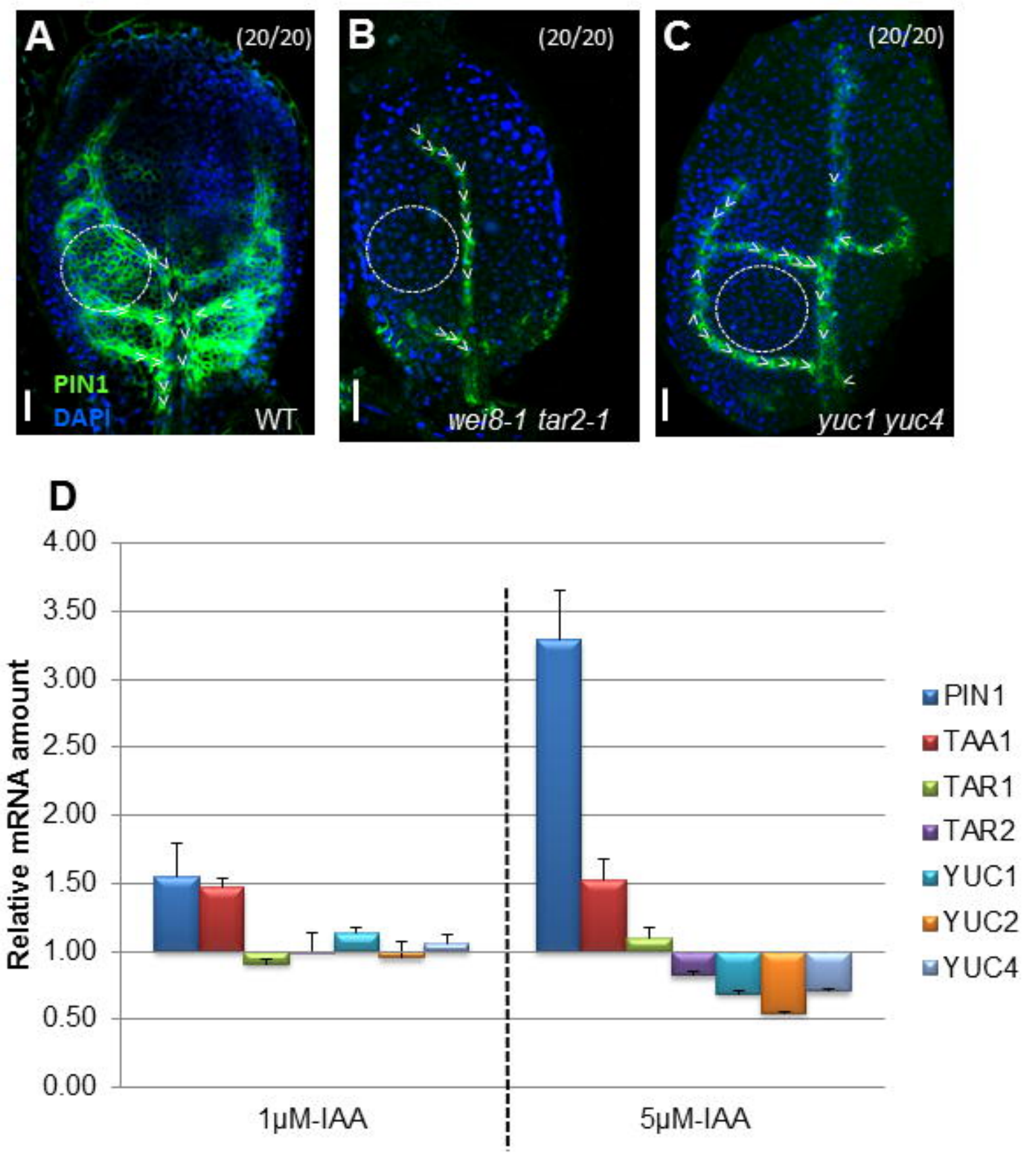

Fig. 6 


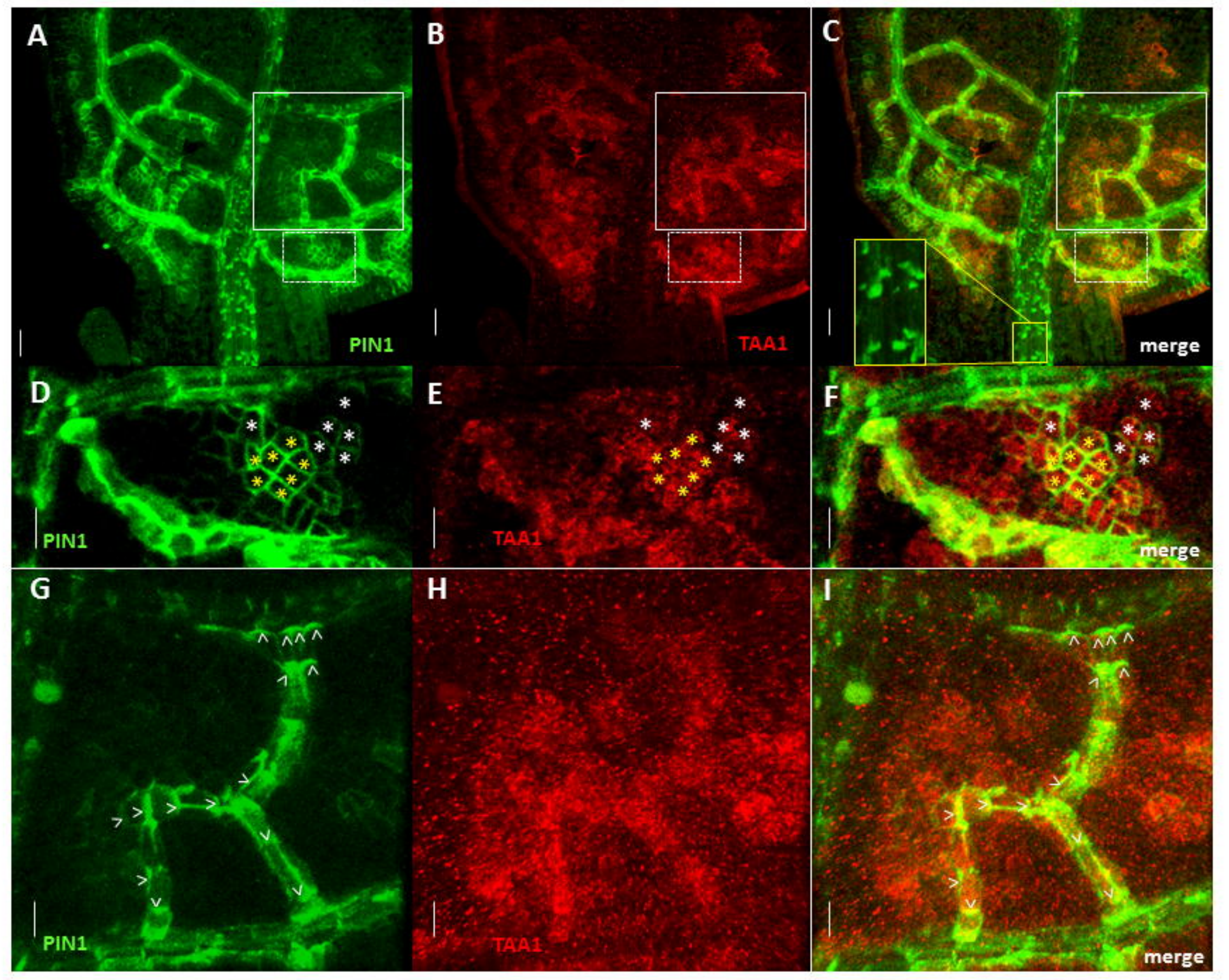

Fig. 7 